\title{
Modular grid-based design concept for fibre reinforced composite shells
}

\author{
E. De Bolster, H. Cuypers, W. P. De Wilde \& J. Wastiels \\ Department of Mechanics of Materials and Constructions, \\ Vrije Universiteit Brussel, Belgium
}

\begin{abstract}
When designing a modular system, two main aspects need to be considered: the aspect of one building stone and the aspect of the overall structure. Both will be studied here as a function of geometry, mechanical behaviour and historical background (architectural design, engineering science, etc.).

The state-of-the-art of design principles, used in architectural designs over the latest decades, will provide the basic knowledge for the generation of a new kind of modular construction. Combining both the artistic point of view (aesthetics) and the engineering point of view (calculations, FEM), a new design concept will be generated: a system with modular hyperbolic paraboloid building stones, connected to one another through hinged connections.
\end{abstract}

Keywords: architecture, modular construction, design concept, hyperbolic paraboloid.

\section{Introduction}

In contemporary architecture, one can notice the tendency towards modular lightweight structures. These designs can easily be assembled and disassembled and they require a minimal amount of materials, which makes this type of design very advantageous. Another tendency that can be observed is the frequent use of freeforms. These designs are usually lightweight as well as aesthetically pleasing. The designs that combine the advantages of modular structures and freeform structures are usually covered with textiles. However, composites (textile reinforced cementmatrix composites, for example) could present a good alternative; especially when used as faces in a sandwich construction. Therefore, it would be interesting to perform a feasibility study of such a modular, freeform 
construction made of sandwich panels with faces of textile reinforced composites.

\section{Hyperbolic paraboloids}

When designing a modular system, two aspects need to be considered: the aspect of one building stone and the aspect of the overall structure, consisting of several building stones. Since a modular system is only as strong as its components (building stones and the connection between them), it is important to select them with care.

A hyperbolic paraboloid (also called: hypar) building stone (see figure 1) has many advantages over other types of building stones. Firstly, a hypar surface can be applied over any foundation shape (rectangular, triangular, circular,...). Secondly, a hypar surface is a curved surface and has therefore better bearing capacities than flat surfaces, because the forces can more easily be introduced in an efficient way (i.e. through higher normal forces and lower bending moments). Due to its anticlastic doubly curved shape, instabilities are less likely to occur: the convex curve will stiffen the behaviour of the concave curve and vice versa (see figure 1, on top).

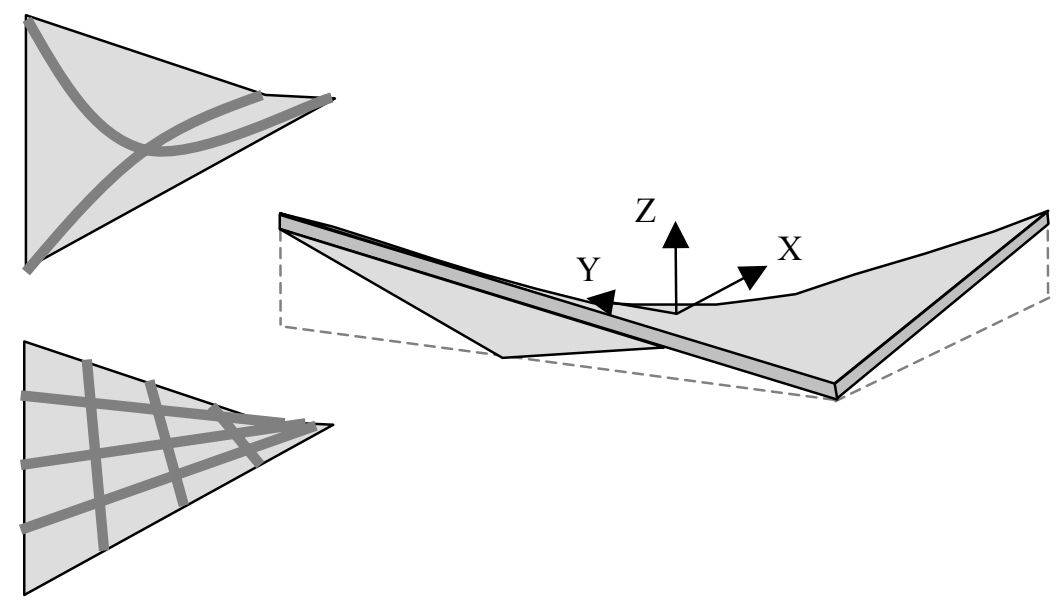

Figure 1: Hyperbolic paraboloid (parabolas, parametric representation and the two distinct independent families of straight lines).

In rectangular coordinates, with the origin in the saddle point of the surface (as indicated in figure 1 , in the middle), the parametric equation of a hypar surface can be given as:

$$
\mathrm{Z}=\mathrm{k} X \mathrm{Y}
$$

where: $\mathrm{k}=\mathrm{a}$ measure for the distortion of the hypar surface. 
Even the fabrication of a hypar surface is not as difficult as one would expect from a three dimensional shape: a hypar surface is a doubly ruled surface and can therefore be formed by two distinct independent families of straight lines (see figure 1, below), which makes the formwork much easier. When manufacturing hypar-sandwich panels with composite faces, an adaptable and reusable cable net could be used as a reconfigurable mould. The net, which is largely based on the ADAPTENT-concept of Hebbelinck [1], then provides an easy, fast, re-usable and economical methodology in fabricating hypar-sandwich panels that are to be used in modular structures (see figures 2 and 3).

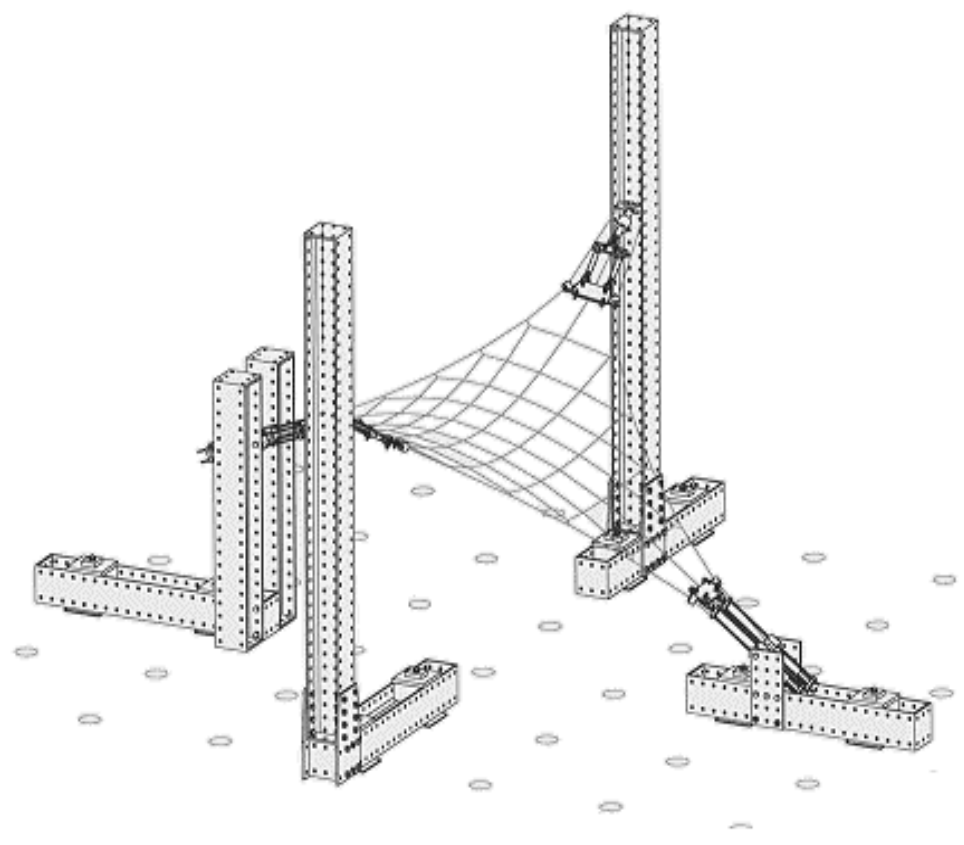

Figure 2: ADAPTENT-concept: asymmetrical hypar surface [1].

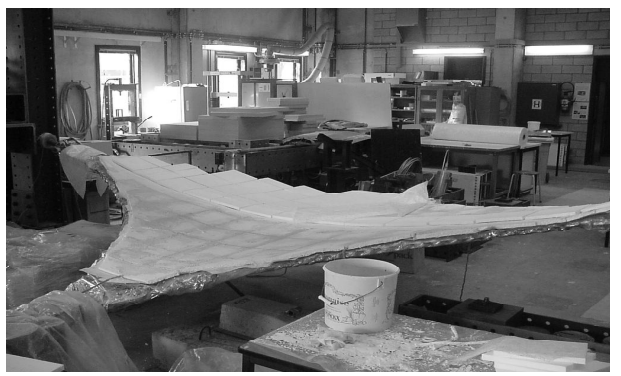

Figure 3: Laminating a hypar sandwich panel on top of the ADAPTENT. 


\section{Design principles}

There are many basic concepts that underlie the field of design. All basic tenets can be grouped into design elements and design principles. They are applicable to all of the visual arts, amongst which architecture. The design elements are the components or objects of the design: a line, a point, a surface, colour, etc. The design principles on the other hand govern the relationship between the design elements, giving them meaning. There are many additional concepts that are related to the principles of design. The grid-concept is one of these techniques: it provides a framework for all basic principles.

\subsection{Grid}

Geometric patterns have always been a fundamental aspect of human culture. In nature, geometric order is rarely found. It is rather man's way to understand his surroundings.

Science uses the grid as a technique to cope with complexity. The grid is the basis for most techniques which sample and quantitatively analyse the 'real world'. In archaeology for example, archaeologists use three dimensional grids as a baseline for complex digs. Excavation takes place within trenches or areas of about 1 metre square, which are located within a site survey 'grid' so that every point on the excavation site can be given a horizontal coordinate. The information, revealed through laborious excavation can then be gathered and ordered in a rather simple manner.

Ecological sampling methods also make use of the grid-method. If one would want to know what kind of plants and animals are in a particular habitat, and how many there are of each species, it is usually impossible to go and count each and every one present. Samples are taken instead. A method of random sampling is used to map the area under survey and to overlay it with a numbered grid. In short, the fundamental technique for filtering the complexity of the observable is based on the 'quadrat' and 'transect': small square or cubic areas (quadrats) are examined in detail along a line or across a grid of evenly distributed sample points (transect).

For reasons difficult to explain, the significance of the grid in modern and contemporary architecture (and art) is just as important.

\subsection{Modern and contemporary architecture}

The grid is one of the oldest architectural design tools. A grid can help a designer control the positions of built and space elements, making the layout task more systematic. Especially in laying out plans for new towns and cities, the use of grids enables the designers to make decisions at the urban scale, nevertheless providing relative freedom at the block and lot scale for individual developers and house designers.

Taking it one step further, the grid can also be applied to a single building. This formal and structural approach to design was seen in the Swiss International Style. The term "International Style" was first used in New York City in 
conjunction with the 1932 Museum of Modern Art exhibition of contemporary European architecture, entitled "The International Style: Architecture Since 1922". The term is often used to refer to all Modern architecture.

According to Philip Johnson and Henry-Russell Hitchcock (who wrote the catalogue that accompanied the exhibition of the International Style), Modern architecture is characterized by three main principals [2]: architecture as volume, regularity and avoidance of applied decoration. The rigid, simple, geometric and asymmetrical style of the International Style arose from the work of Walter Gropius, Ludwig Mies van der Rohe and of course Le Corbusier and lived on in the work of - for example - Skidmore, Owings \& Merrill. Many architects retained the earlier mentioned modernist philosophies, aiming to integrate modern technology and formal elements derived from the basic grid. Mies used minimalistic, linear forms, evoking the language of the idealistic International Style philosophy, called "less is more". He embraced the glass and steel skyscraper that showed its building materials as a form of ornamentation. His buildings were carefully laid out on a grid, totally embracing the concept of regularity. This careful grid system lined up everything including the tiles in the plaza and even the lighting in the ceiling of the lobby. One of his well known accomplishments is the Barcelona Pavilion in 1929. The grid-system is undeniably present.

The ideal modernistic point of view became so stringent that a rupture between architects and this architectural style was inevitable. Postmodernism rebelled against the purity and rigidity of forms with lots of excess and exuberance. Nowadays, in the rise of spirituality and fundamentalism however, architecture has thrown off the ornamentation of postmodernism and returned to some of the values of the International Style. In his book "Supermodernism: Architecture in the Age of Globalization" [3], Hans Ibelings defines the postpostmodernism as a tech-inspired aesthetic movement that reacts against postmodernism and adopts the philosophy of computer product design. Architects are drawn towards the idea of "tabula rasa"; the idea that each new design starts as a pure, empty plane. Structures appear portable and therefore disconnected from their surroundings. Similar to a computer, all the details are inside, while exteriors are neutral and unassuming. High-tech knowledge is used to create spaces that are valued for their visual and spatial sensations only. Since high-tech architecture strongly relates to science, the same techniques of handling complexity are likely to be used. It seems evident that - yet again - the grid-system is a widely-spread designing tool.

\section{Overall design}

Many have said grid-systems can suffocate creativity, as can modularity. However, this doesn't have to be the case. Grid-systems can facilitate creativity by providing a framework and some predefined answers to questions such as "What span to use?", "Which module to use?", etc. The grid simplifies decisionmaking by limiting the placement of elements to certain places, but if the grid 
doesn't permit a sufficiently rich range of variation, the designer must react and redesign it.

\subsection{Design concept}

In this paper a design concept is formulated in which a grid is used as an integration system (through hinged line connections) of the individual hypar surfaces in the overall "roof"-design. A two-dimensional grid is formed that defines the horizontal coordinates of the four corner points of each hypar surface. The vertical coordinate of the corner points depends on the formal design of the overall structure and is thus insofar independent of the grid. Once the position of four non-coplanar corner points are chosen, a hypar surface can be created. With this systematic method, a variety of shapes can be made modular in five steps:

1. Choose a mathematical function $\mathrm{z}=\mathrm{f}(\mathrm{x}, \mathrm{y})$, representing the formal aesthetics you - as designer - wish.

2. Choose/note the dimensions of the horizontal rectangular base that should be covered.

3. Divide this base in a number of elements (each element having the same dimensions in the $\mathrm{x}$ - and $\mathrm{y}$-direction).

4. Calculate the $\mathrm{x}$ - en $\mathrm{y}$-coordinates of each corner point (on the base).

5. Use these coordinates and the mathematical equation of the threedimensional function to calculate the $\mathrm{z}$-coordinate of each corner point.

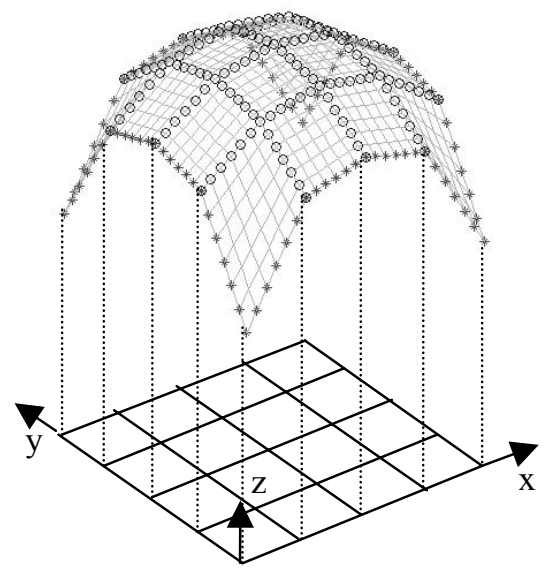

Figure 4: Generating a modular hypar-system, based on a grid.

The two-dimensional horizontal grid not only defines the overall design, but also facilitates the prefabrication of each hypar surface (see section 2). In figure 4 , it was chosen to work with a single regular grid to which each building stone is related. 
Since any mathematical function can be chosen as initial aesthetic design, a variety of freeform constructions can be created without the fear of losing oneself in an infinite amount of choices. Due to the grid and the specific shape of the basic building stone, one is better capable of handling the complexity of freeform design: a framework has been set that still leaves room for ones own creativity and originality.

\subsection{Modularity science}

The abovementioned design idea has similarities with the finite element method. In FEM, a complex object is simplified by dividing it in areas (finite elements) and reducing each finite element to his corner points. Calculations of the stresses are done in specific, discrete Gauss points. In the presented modular design system, an overall shape is divided into pieces according to a horizontal grid and approximated by the discrete corner points of each piece. The design is dictated by the hypar surfaces that are drawn through the discrete points.

The similarities between the finite element method and designing method are clarified in figure 5: a geodesic dome of Buckminster Fuller is shown. An aluminium sphere with radius of $34.14 \mathrm{~m}$ is used as roof for the former aviation museum at the airport of Schiphol in the Netherlands. Starting from the formal design (figure 5, top left), calculation methods are used to "mesh" the object under study (figure 5, middle right). Each "mesh" is then extracted from the overall object and studied individually: the module is born (figure 5, bottom left) to which all calculations are related.
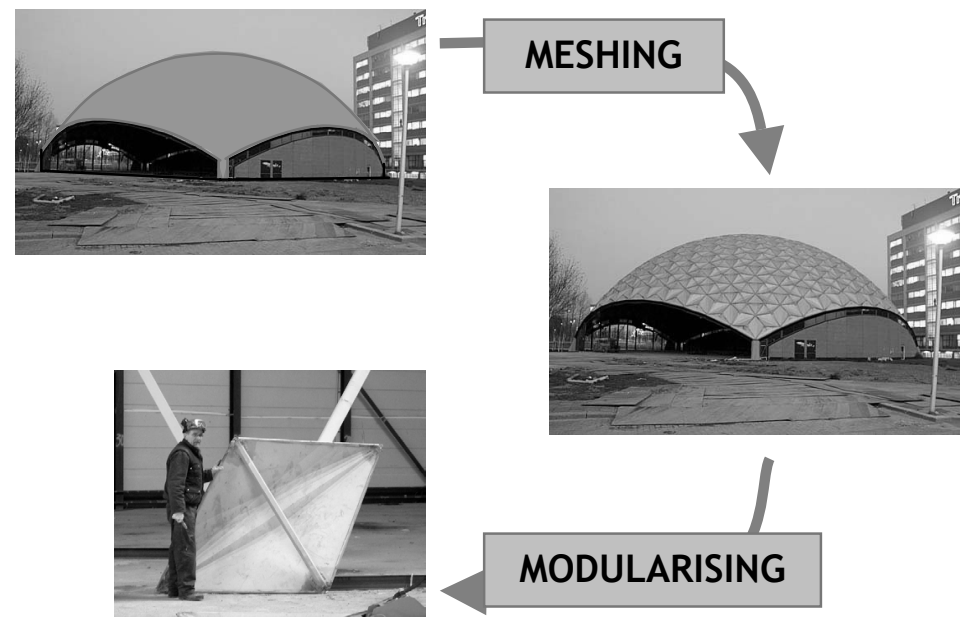

\section{MODULARISING}

Figure 5: From global concept to discrete solutions (National Aviation museum Aviodome-Schiphol, Buckminster Fuller, 1971). 
As with FEM, the approximation of the original object is best when the griddimensions (or in case of FEM: the mesh-sizes) reduce to zero. Similar to FEM, it is expected that with a certain grid(mesh)-size a convergence can be reached. This convergence limits the amount of hypar surfaces that needs to be created and therefore limits the time needed for design, fabrication and erection of the modular structure.

In the given analogy between finite elements and the modular design system under study has a slight discrepancy at the end. When the division of an arbitrary object in finite elements has occurred, calculation is performed in discrete points. When the division of an arbitrary roof design in modular elements has occurred, calculation is performed on each module. In other words, in finite elements calculation is done in discrete points, while in the presented modular designconcept calculation is said to be done on a surface. In reality, calculation of each module will be done through the use of finite elements. The modular hypar surface building stone becomes the arbitrary object that will be divided in a certain amount of finite elements and calculated in the discrete Gauss points of each finite element (see figure 6).

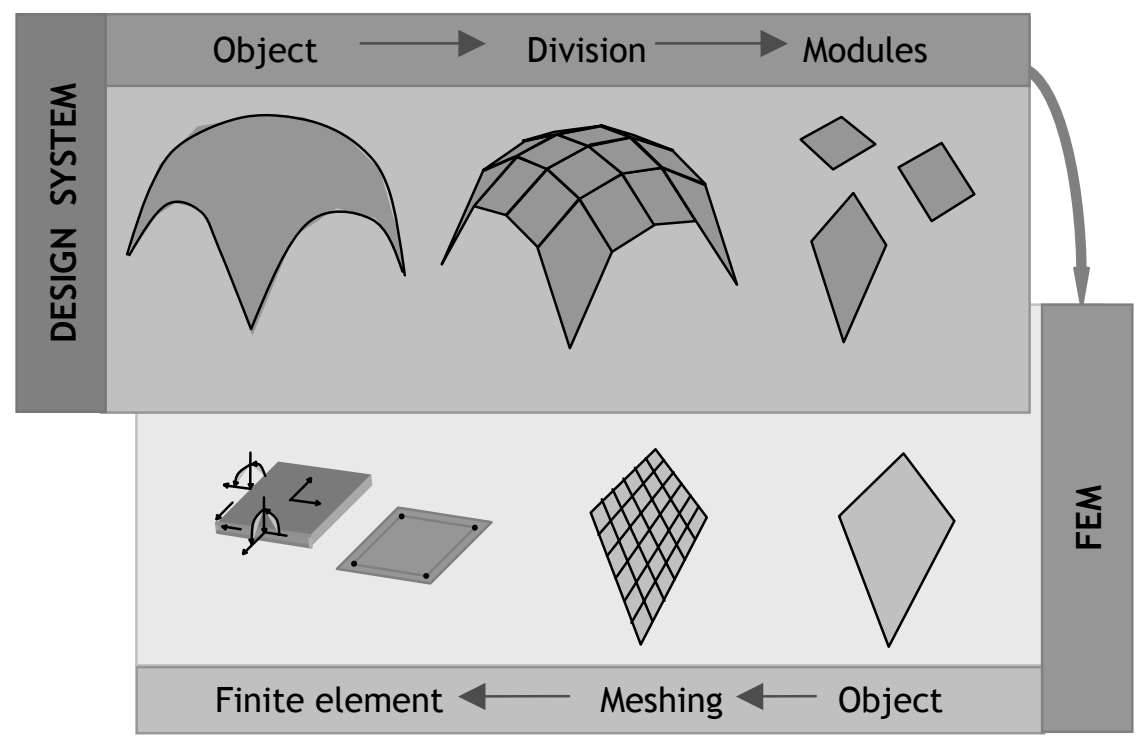

Figure 6: Level-wise calculation of an arbitrary design shape.

\section{Conclusion}

The grid-system has always been an important design principle: not only in science, art, etc, but also in architecture. Modern and contemporary architecture have turned it into an undeniable part of building concepts. The evolution of scientific knowledge has turned mankind's urge of controlling his surroundings, and thus the grid, into an unmistakable part of building realisations. 
The presented design concept of modular hypar surface-structures originates from the combination of a two-dimensional, horizontal grid and a chosen, aesthetically pleasing, three-dimensional design-shape. The concept shows a lot of similarities with the finite element method: approximating continuous objects (the overall design shape) by discrete objects (the modular building stones, which are hyperbolic paraboloid surfaces). On a second level, it even makes use of the finite element method: calculations are done in discrete points and no longer on continuous surfaces.

\section{Acknowledgment}

Financial support from the Institute for the Promotion and Innovation by Science and Technology in Flanders ("IWT-Vlaanderen") is gratefully acknowledged.

\section{References}

[1] Hebbelinck, S., A generating system for temporary, adaptable and reusable nets and tensile structures, PhD-thesis Vrije Universiteit Brussel, Belgium, 2002

[2] Leland, M., About the modern Style, http://www.michael.leland.name/ modern/index.html

[3] Ibelings, H., Supermodernism: architecture in the Age of Globalization, Nai Publishers, Rotterdam, 1998 